\title{
A Multidisciplinary Approach in Pharmacovigilance Awareness: ISoP Egypt Chapter's MedSafetyWeek Experience
}

\author{
Hadir Rostom ${ }^{1,2}$ (D) Mohamed A. Elhawary, ${ }^{1,3} \cdot$ Islam N. Ali ${ }^{3,4}$ (D \\ Accepted: 8 August 2021 / Published online: 23 August 2021 \\ (C) The Author(s), under exclusive licence to Springer Nature Switzerland AG 2021
}

\section{Introduction}

Pharmacovigilance (PV) is more important than ever, with worldwide clinical trials taking place and several older medicinal products being repurposed. The underreporting of adverse drug reactions (ADRs) resulted in an underestimation of their frequency and severity [1]. In light of this, the Uppsala Monitoring Center (UMC), WHO Collaborating Center for International Drug Monitoring, coordinates 'MedSafetyWeek', an annual virtual campaign on social media for healthcare professionals (HCPs) and public alike, during which an animated series featuring humorous characters whose medicinal product misuse results in long-lasting health consequences is displayed [2]. Raising the awareness of PV and medication safety during MedSafetyWeek is essential and will certainly help in improving the reporting rates.

$\mathrm{PV}$ and ADR reporting practices are relatively new for Egypt. Addressing medication safety challenges demands multidisciplinary collaborations that bring together the tools, efforts and resources of different professionals to assess and manage ADRs jointly. Safety monitoring of medicinal products in all its aspects is a challenge increasingly taken up by multilateral organizations and institutions, including hospitals, academia, student organizations, community pharmacies, pharmaceutical companies, medicine regulatory authorities and other independent institutions.

Islam N. Ali

islam.nabil.ali@pharma.asu.edu.eg

1 International Society of Pharmacovigilance (ISoP), Egypt Chapter, Cairo, Egypt

2 Faculty of Pharmacy, MSA University, 6th of October City, Giza, Egypt

3 Faculty of Pharmacy, Ain Shams University, Cairo, Egypt

4 University of Glasgow, Glasgow, Scotland, UK

\section{International Society of Pharmacovigilance (ISoP) Egypt Chapter}

The Egyptian Chapter of the International Society of Pharmacovigilance (ISoP) was launched in early 2020 amid the current coronavirus 2019 (COVID-19) outbreak, with the aim of augmenting PV practice [3]. As such, the primary objectives of the ISoP Egypt Chapter include increasing caregivers and patients' awareness about medication safety, as well as shedding light on ADRs and other safety incidents in Egypt. Other objectives include enriching the PV practice within pharmaceutical companies, fostering risk management practice, conducting PV studies, encouraging PV education at all levels, supporting the integration of PV in public health programs, and providing trainings in $\mathrm{PV}$ and medication safety [4].

Once established, the ISoP Egypt Chapter aimed at assembling stakeholders in an integrated approach to encourage partnerships and multidisciplinary knowledge sharing by carrying out several activities involving interactive awareness materials on social media, research topics relevant to medication safety information, and online training sessions. Such cooperation is promoted in the context of keeping the Egyptian Chapter of ISoP at the forefront of medication safety practices through the introduction of new ideas and applying the best tools to present innovative projects. All of these key benefits of collaboration are described below.

1. Provide insight into the healthcare system in the context of a broader framework.

2. Build relationships that involve a level of trust with key stakeholders (patients and HCPs).

3. Implement resources in the community to recruit new allies.

4. Set-up an effective safety monitoring system, backed up with risk minimization measures. 
5. Support local volunteering initiatives and work under a common protocol.

6. Adopt a systematic reporting method to confirm best practices for stakeholder engagement.

It is worth mentioning extended collaborative projects conducted between the ISoP Egypt Chapter and the European Union Pharmacovigilance and Pharmacoepidemiology (Eu2P) Master of Science (MSc) degree. Eu2P offers specialization that fulfills professional needs in scientific research and medicine risk communication along with graduation of a new batch able to develop PV systems capable of achieving high standards of public health protection in Egypt.

\section{Multidisciplinary Approach in Pharmacovigilance Awareness}

Collaborations in global health frequently occur through partnerships in which organizations share responsibilities and decision-making processes, with the objective of collectively and more effectively tackling a common challenge. Having said that, the fifth annual meeting of the MedSafetyWeek campaign, which took place over the course of 1 week between 2 and 8 November 2020 under the theme of 'Every Report Counts!', was an indispensable opportunity to meet ISoP Egypt Chapter priorities and appreciate the frontline healthcare workers who are performing their work under particularly extreme pressure throughout this ongoing unprecedented situation.

Accordingly, the ISoP Egypt Chapter decided to take the campaign a bit further by enabling the engagement of targeted stakeholders, ranging from organizations such as hospitals, academia, community pharmacies and pharmaceutical companies to individuals such as pharmacists, pharmacy students, and PV staff at pharmaceutical companies.

The extent to which key stakeholders-patients and HCPs - will be able to engage in primary PV activities is an important enabler to effectively improve and optimize the functioning of the PV system, as well as promoting deeper levels of participatory involvement in conveying a particular campaign message. Their role extends to providing information by means of reporting suspected ADRs and amending their attitudes in risk minimization interventions. When effectively implemented, the open dialogue meetings will allow stakeholders to distinguish which activity is the most effective and to identify a series of enablers along with their corresponding barriers for the sake of PV consolidation throughout the healthcare system.

\section{Shaping the Campaign Field Activities and Stakeholders' Involvement}

The ISoP Egypt Chapter believed it was important to cultivate the culture of ADR reporting and boost PV awareness in general by getting stakeholders actively engaged in campaign message delivery. In this sense, a flexible and diverse proposal of stakeholder engagement was carried out to suit different audiences by pursuing contacts through one or more of the following:

1. Hosting scientific sessions dealing with PV roles of HCPs (mainly in hospitals).

2. Conducting field visits to community pharmacies and hospitals.

3. Disseminating campaign flyers (essentially in community pharmacies together with the dispensed medication).

4. Displaying campaign posters, including a brief message in Arabic about the significance of reporting, along with campaign characters and a QR code for further details on social media activities.

5. Hosting a booth at the campaign, while approaching patients, HCPs, and students.

6. Allowing students of different medical specialties to join and participate in the ISoP Egypt Chapter activities.

7. Printing campaign flyers and posters.

Printed campaign awareness materials were conceived and designed based on the concept of similar characters developed and used by UMC along with additional follow-up social media calls for more PV details. Social media outreach strategy proved successful in providing media coverage for the field activities and reaching the stage of scaling up the intended effect. Relevant posts of our campaign coverage have recorded a relatively high engagement rate and have been perceived as an acknowledgment of participants' hard work during their field research activities.

Typically, stakeholders such as pharmaceutical industry, pharmacy chains, hospitals, academia and other organizations can help add various strengths and resources by introducing their own organizational cultures and regulations. In this regard, the collaboration between the ISoP Egypt Chapter and various stakeholders will build a culture of continuous feedback mechanism that acts as a platform through which the true value of effective stakeholder engagement is identified. 


\section{Diversity of the ISoP Egypt Chapter Target Stakeholders}

In order to guarantee reliable PV training sessions, individual interviews and group discussions amid the MedSafetyWeek 2020 campaign, it was essential to capture different types of target stakeholders in terms of direct interactions in medical settings and related expectations for the Egyptian Chapter of ISoP.

At this point, reporting of ADRs in Egypt remains voluntary for health workers. Regardless, most of them agreed that the existence of an ADR database system to report any medication safety concerns should be mandatory in all clinical settings and that a clinical pharmacist would be held accountable for such a system. However, the role of HCPs is substantial, not only in detecting and reporting ADRs but also in considering the risk minimization measures associated with their clinical practice.

Almost all participants expressed a positive view of the concept of risk minimization measures, including the distribution of educational materials by pharmaceutical companies as an integral part of their PV practices, and inferring that those companies are compliant with national authority guidelines and therefore seem to be trustworthy. Some pharmaceutical companies delegated their PV specialists in an effort to empower caregivers' awareness about their own roles and responsibilities accordingly.

Visits to community and hospital pharmacies formed an integral part of our campaign field activities. Communitybased pharmacies in particular serve the public directly, and thus pharmacists contribute to a great extent in ADR evaluation, monitoring and promotion of the optimum medicine consumption. Almost all of them have been sporadically approached by some patients regarding complaints that were associated with underlying ADRs. The minority who already had a prior notion of reporting processes of ADRs would also have a negative perception about PV in terms of being an additional burden, an aspect for which the current working systems may be amended to incorporate ADR reporting systems into their professional duties.

As the concept of shared decision making is becoming more and more mainstream, direct involvement of patients in the reporting of ADRs provides a different insight into drug safety. Setting up booths at community pharmacies and hospitals helped in reaching out to patients, many of whom were in favor of direct reporting instead of filling out the reporting form themselves.

Among participants, pharmacy students were the most energetic, not to mention their interactive engagement on social media. Their involvement went beyond that of being a cluster in the target audience by actively participating in field activities, including paying visits to community pharmacies and conducting PV awareness sessions aimed at other students and academic staff, both medical and non-medical. It should also be mentioned that they demonstrated a willingness to learn more about PV during their college education. In this context, new educational interventions are being studied to promote their effective participation in reporting activities and, above all, to prevent ADRs in the community. Finally, we have been approached by several student organizations in different universities to become involved in the training of specialized PV personnel in both clinical and community settings.

\section{Conclusion}

Partnerships between the ISoP Egypt Chapter and other organizations emerged as an influential aspect of systemic shift in the patient safety culture by means of addressing certain challenges with regard to implementing PV in Egypt. A diversity of approaches involving different stakeholders has added value to the designated campaign and one of its critical success factors. ISoP's Egypt Chapter took the initiative and conducted the first-ever large-scale campaign throughout Egypt for the purposes of integrating social media and field activities, as well as engaging a variety of key stakeholders.

Acknowledgements The authors acknowledge the assistance of Dr Katarina Ilic, ISoP Comms Leader, and Deirdre McCarthy, ISoP Secretary-General, for reviewing the manuscript. The authors also wish to thank all supporting and collaborating organizations, along with those who gave time to participate in our campaign. Special thanks go to October University for Modern Sciences and Arts (MSA University), El Ezaby Pharmacy, Dar Al Fouad Hospital, Nozha International Hospital in Cairo, Eli Lilly Egypt and Medical Union Pharmaceuticals for their continued support and cooperation.

\section{Declarations}

Funding The authors received no specific funding for this work.

Conflict of interest Hadir Rostom, Mohamed A. Elhawary and Islam $\mathrm{N}$. Ali have no conflicts of interest related to the content of this manuscript.

Ethics approval Not applicable because this article does not contain any studies with human or animal subjects.

Consent to participate Not applicable.

Consent for publication Not applicable.

Availability of data and material Data sharing is not applicable to this article.

Code availability Not applicable. 
Author contributions Conception: HR and MAE. Drafting, writing, review, and editing of the manuscript: INA and MAE. Critical revision: HR, INA and MAE. All authors read and approved the final version.

\section{References}

1. Lopez-Gonzalez E, Herdeiro MT, Figueiras A. Determinants of under-reporting of adverse drug reactions: a systematic review.
Drug Saf. 2009;32(1):19-31. https://doi.org/10.2165/00002018200932010-00002.

2. Uppsala Monitoring Centre. Medication Safety Week. www.whoumc.org/medsafetyweek/.

3. Harrison-Woolrych M. A new president's vision for international pharmacovigilance. Drug Saf. 2020;43(2):79-81. https://doi.org/ 10.1007/s40264-019-00902-6.

4. International Society of Pharmacovigilance. Egypt Chapter. https://isoponline.org/chapters/egypt/. 